\title{
Editorials
}

\section{What's a nice patient like you doing with a complication like this? Diagnosis, prognosis and prevention of spinal hematoma}

\author{
Terese T. Horlocker MD
}

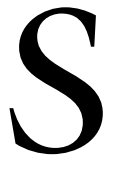

PINAL hematoma is a rare and potentially catastrophic complication of spinal or epidural anesthesia. The actual incidence of neurologic dysfunction resulting from hemorrhagic complications associated with central neural blockade is unknown. In an extensive review of the literature, Tryba identified 13 cases of spinal hematoma following 850,000 epidural anesthetics and seven cases among 650,000 spinal techniques. ${ }^{1}$ Based on these observations the calculated incidence was approximated to be less than 1 in 150,000 epidural and less than 1 in 220,000 spinal anesthetics. ${ }^{1}$ Since these estimates represented the upper limit of the $95 \%$ confidence interval, the overall frequency is much less. In addition, patient characteristics and anesthetic variables associated with an increased risk of spinal bleeding, compared to these baseline values, have been identified by case reports and clinical series. ${ }^{2-4}$ For example, the risk associated with an indwelling epidural catheter in the presence of twice daily administration of low molecular weight heparin (LMWH) has been estimated to be one in 3,100 patients. ${ }^{5}$ However, while it is useful to identify patient populations at risk, even more crucial are management techniques which facilitate the detection and evaluation of new perioperative neurologic deficits.

In this issue of the Canadian Journal of Anesthesia, Schwarz et al. ${ }^{6}$ report a spinal hematoma associated with standard $s c$ heparin thromboprophylaxis in the presence of epidural catheterization. The patient made a full neurologic recovery, despite lack of surgical intervention. The title highlights the unique aspects of the case - patient age is a potential risk factor and spontaneous neurologic recovery may occur despite significant deficits. The title also demonstrates the enduring perception that sensory and motor deficits are atypical presenting symptoms of spinal hematoma. This misconception continues to delay intervention and impact neurologic outcome.

The introduction of new anticoagulants and antiplatelet agents, the complexity of balancing thromboembolic with hemorrhagic complications and the evolving indications for regional anesthesia/analgesia necessitate an individualized approach. Hopefully, the ensuing analysis of ongoing trends and controversies comprising "everything you wanted to know about spinal hematoma, but never had the chance to ask", will assist with management decisions.

\section{What is the frequency of spontaneous spinal hematoma?}

Spinal hematoma in the absence of trauma or anticoagulant therapy rarely occurs. However, major spontaneous hemorrhagic complications, such as intracranial, intraspinal, intraocular, mediastinal or retroperitoneal bleeding, have been reported after antithrombotic and thrombolytic therapy. Risk factors include the intensity of the anticoagulant effect, increased age, female gender, history of gastrointestinal bleeding, concomitant aspirin use, and length of therapy. ${ }^{7}$ During warfarin therapy, an international normalized ratio (INR) of 2.0 to 3.0 is associated with a low risk of bleeding: $<3 \%$ during a three-month treatment period. Higher intensity regimens (INR > 4) are associated with a significantly greater risk of bleeding $(7 \%)$. The incidence of hemorrhagic complications during therapeutic anticoagulation with standard heparin, as well as LMWH, is < $3 \%{ }^{7}$ Thrombolytic therapy represents the greatest risk of bleeding; between $6 \%$ and $30 \%$ of patients. ${ }^{8}$ Patients who require preoperative anticoagulant therapy, such as those with a history of atrial fibrillation or cardiac valve

From the Department of Anesthesiology, Mayo Clinic, Rochester, Minnesota, USA.

Address correspondence to: Dr. Terese T. Horlocker, Department of Anesthesiology, Mayo Clinic, Rochester, MN 55905, USA. Phone: 507-284-9694; Fax: 507-284-0120; E-mail: horlocker.terese@mayo.edu 
replacement, are not ideal candidates for neuraxial techniques. Often the anticoagulant effect is only partially reversed to avoid thrombotic complications and/or they are aggressively anticoagulated postoperatively. Normal hemostasis for needle placement/catheter removal is present only for a short interval.

\section{Which patient factors are associated with a significant risk of spinal hematoma?}

The patient factors for spinal hematoma are similar to those for spontaneous bleeding with antithrombotic therapy - increased age and female gender. Decreased weight and concomitant hepatic or renal disease may also exaggerate the anticoagulant response and theoretically increase the risk. In a series of 40 spinal hematomas associated with LMWH thromboprophylaxis, $75 \%$ of the patients were elderly women. ${ }^{3}$ LMWH dosing is not weight adjusted and the anticoagulant effect of LMWH is not monitored, due to reliable bioavailability. Furthermore, although renal insufficiency is known to prolong and increase the anti-Xa effect, a baseline serum creatinine was not always measured prior to initiation of LMWH thromboprophylaxis. These factors may have contributed to the number of spinal hematomas. Warfarin pharmacology is also significantly affected by patient age, diet and activity. The wide variability in patient response to warfarin necessitates frequent monitoring. Obviously, patient factors are not "controllable", but should be considered when selecting a regional technique and intensity of neurologic monitoring perioperatively.

\section{What is the source of the bleeding and how much blood must accumulate before spinal cord ischemia occurs?}

The majority of spinal hematomas occur in the epidural space because of the prominent venous plexus. Conversely, subarachnoid bleeding causing neurologic compromise is seldom reported because of the diluting effect of cerebrospinal fluid and lack of major blood vessels. The actual source of the bleeding (arterial $v s$ venous) is controversial. Bleeding from an arterial source should accumulate rapidly and cause neural ischemia soon after vessel trauma. However, most spinal hematomas become symptomatic several days after needle/catheter placement, not immediately postoperatively, suggesting the bleeding is not arterial. On the other hand, a venous source would accumulate more slowly, but theoretically would tamponade prior to overcoming spinal cord perfusion pressure. Thus, neither model entirely represents the clinical scenario. The volume of blood required to cause cord ischemia also varies, and is affected by the site of bleeding (the cauda equina is relatively resistant, while the watershed areas of the cord are more easily compromised), the presence of vertebral column abnormalities, and the rapidity in which the blood accumulates. It is interesting to note that several of the LMWH hematomas involved less blood than that typically injected during the performance of an epidural blood patch. ${ }^{3}$

\section{What is the usual timing of onset of neurologic symptoms?}

The onset of symptoms immediately postoperatively is uncommon; it is rare for a spinal hematoma to present as a "prolonged" neuraxial block. In one series, the median time interval between initiation of LMWH therapy and neurologic dysfunction was three days, whereas the time between onset of symptoms and laminectomy was more than $24 \mathrm{hr}^{3}{ }^{3}$ Less than one-third of patients reported fair or good neurologic recovery. These results are consistent with those of Vandermeulen et al., ${ }^{2}$ who reported the time to progress from new neurologic deficits to complete paralysis was approximately $15 \mathrm{hr}$. In addition, it was noted that if more than eight hours was allowed to elapse between the development of paralysis and surgical intervention, complete neurologic recovery was unlikely. ${ }^{2}$

\section{At what point should radiographic imaging be per- formed and/or the neurosurgeon consulted?}

The differential diagnosis of new or progressive postoperative neurologic symptoms includes surgical neuropraxia, prolonged/exaggerated neuraxial block, anterior spinal artery syndrome, exacerbation of a preexisting neurologic disorder, and presentation of a previously undiagnosed neurologic condition, as well as spinal hematoma. Evaluation is focused on identification of reversible/treatable causes. Therefore, any new or progressive neurologic symptoms occurring in the presence of epidural analgesia warrant immediate discontinuation of the infusion (with the catheter left in $s i t u$ ) to rule out any contribution from the local anesthetic or volume effect. If the epidural is the etiology of the deficits, a prompt return of function should be noted. Since neurologic outcome is linked to early diagnosis and intervention, it is critical to obtain radiographic imaging, preferably magnetic resonance imaging, as soon as possible. Consultation with a neurosurgeon should also occur as soon as possible to determine the urgency of surgery. Interestingly, not all spinal hematomas are treated with emergency laminectomy; spontaneous resolution of deficits has been reported. ${ }^{6,9}$ However, the decision to observe vs surgically intervene is a neurosurgical one. 


\section{Has spinal hematoma been reported in the parturient} population?

Since pregnancy and the immediate postpartum interval are associated with a hypercoagulable state, it is often assumed that parturients are not at risk for spinal hematoma. However, in the series by Vandermeulen $e t$ al., ${ }^{2}$ five of 61 spinal hematomas involved parturients. In two cases, a clotting disorder (thrombocytopenia, preeclampsia) was present. One parturient had a previously undiagnosed epidural ependymoma. No risk factors were reported in the remaining two patients. To date, there have been no spinal hematomas associated with peripartum antithrombotic therapy. However, there is no large series documenting the safety of neuraxial block in the presence of the therapeutic levels of anticoagulation required among this patient population. Therefore the relative risk is unknown.

\section{Compared to the surgical and obstetrical popula- tions, do ambulatory pain clinic patients warrant additional consideration?}

Several investigations and case reports suggest that patients undergoing blocks for chronic pain therapy are at increased risk for neurologic complications. ${ }^{10,11}$ In a review of the American Society of Anesthesiologists Closed Claims Project, Cheney et al. ${ }^{10}$ reported that spinal cord injuries were the leading cause of claims for nerve injury in the 1990s and that a major factor associated with spinal cord injury was block for chronic pain management, including epidural steroid injection. There were no spinal hematomas in a series of 1,214 patients undergoing epidural steroid injection, including $383(32 \%)$ who were receiving antiplatelet medications. ${ }^{12}$ However, there were 42 patients with new neurologic symptoms or worsening of preexisting symptoms that persisted for more than $24 \mathrm{hr}$ after injection. Median duration of the symptoms was three days. It was speculated that either a pressure (ischemic) effect of the injection or an inflammatory response to one or more of the components contributed to these neurologic deficits. It was recommended that patients be instructed to seek medical attention should evidence of neurologic compromise occur, since these are the presenting symptoms of spinal hematoma.

\section{What are the options for patients who emergently require anticoagulation or thrombolytic therapy?}

Patients who have recently undergone neuraxial anesthesia and require emergent anticoagulation for limb ischemia, acute coronary syndrome, or deep venous thrombosis/pulmonary embolism represent a conundrum for the anesthesiologist. Ideally, the patient should be queried prior to antithrombotic or throm- bolytic therapy for a recent history of lumbar puncture, spinal or epidural anesthesia, or epidural steroid injection to allow appropriate management and monitoring. The decision to maintain or remove an existing neuraxial catheter is based on the degree/duration of antithrombotic therapy. Since combination therapy (antiplatelet medications with heparin and/or thrombolytics) represents a greater risk of bleeding, treatment options with the least impact on coagulation should be considered. ${ }^{7,8}$ Unfortunately, while the anesthesia community is well aware of the potential for spinal bleeding in this patient population, other specialties have only recently become cognizant of the risk. As a result, the anesthesiologist may not be notified until after establishment of therapeutic anticoagulation. Under these circumstances, a consensus on when hemostasis may be restored to allow catheter removal must be achieved, balancing the relative risks of hemorrhage and thrombosis. Complete reversal of the anticoagulant effect may not be feasible due to the risk of thromboembolic complications. Most importantly, ongoing efforts to educate clinicians responsible for administering hemostasis-altering medications are critical.

\section{New antithrombotic agents are introduced continual-} ly. What is an appropriate strategy?

New antithrombotic drugs which target various steps in the hemostatic system, such as inhibiting platelet aggregation, blocking coagulation factors, or enhancing fibrinolysis are continually under development. The most extensively studied are antagonists of specific platelet receptors and direct thrombin inhibitors. Many of these antithrombotic agents have prolonged half-lives and are difficult to reverse without the administration of blood components. Furthermore, since the risk of thromboembolic complications is decreased with intraoperative or early postoperative initiation of antithrombotic therapy, it is likely that the new pharmacologic therapies will utilize these principles. Unfortunately, early postoperative dosing is associated with surgical (and often anesthesia-related) bleeding. This has been noted with standard heparin, LMWH, and fondaparinux. ${ }^{3,7,13}$

It will probably not be possible to evaluate the risk of spinal hematoma based on data from the phase II and III clinical trials of new agents. Pharmaceutical companies and the Food and Drug Administration construct methodologies to minimize the potential for this catastrophic complication. For example, during the clinical testing of fondaparinux, the patient was excluded from the trial if neuraxial block was traumatic or more than a single needle pass was required. ${ }^{13}$ Until a large body of literature is available to document the safety of neurax- 
ial techniques in patients treated with antithrombotic medications such as fondaparinux, the decision to perform neuraxial blockade should consider the frequency and severity of surgical bleeding (as a surrogate for spinal bleeding), the conditions under which neuraxial block was performed during the clinical trials, and the risk of alternative anesthetic techniques. Importantly, the prolonged effect of the investigational antithrombotic agents will make it difficult to remove indwelling catheters during normal hemostasis. It is likely that indwelling neuraxial catheters will be removed prior to initiation of thromboprophylaxis.

\section{Do continuous peripheral blocks represent "mini- mum risk"?}

Although spinal hematoma is the most significant hemorrhagic complication of regional anesthesia due to the catastrophic nature of bleeding into a fixed and noncompressible space, the associated risk following plexus and peripheral techniques remains undefined. There are no investigations which examine the frequency and severity of hemorrhagic complications following plexus or peripheral blockade in anticoagulated patients. However, few reports of serious complications following neurovascular sheath cannulation for surgical, radiological, or cardiac indications have been reported. The few reported cases of major bleeding associated with non-neuraxial techniques occurred after psoas compartment or lumbar sympathetic blockade; neurologic compromise was not always reported. ${ }^{14-16}$ These cases suggest that significant blood loss (resulting in transfusion or even death), rather than neural deficits, may be the most serious complication of non-neuraxial regional techniques in the anticoagulated patient. Given the paucity of information, it is impossible to make definitive recommendations. Conservatively, the consensus statements on neuraxial anesthesia and anticoagulation may be applied to plexus and peripheral techniques. ${ }^{17}$ However, this may be more restrictive than necessary for techniques involving superficial and compressible vasculature.

In summary, risk factors for spinal hematoma include patient, pharmacologic and regional anesthetic variables. However, it must be remembered that identification of risk factors and establishment of guidelines will not completely eliminate the complication of spinal hematoma. In Vandermeulen's series, although $87 \%$ of patients had a hemostatic abnormality or difficulty with needle puncture, $13 \%$ had no identifiable risk factor. ${ }^{2}$ Vigilance in monitoring is critical to allow early evaluation of neurologic dysfunction and prompt intervention. We must focus not only on the prevention of spinal hematoma, but also on the optimization of neurologic outcome.

\section{Qu'est-ce qu'un patient comme vous fait avec une complication comme celle-là ? Diagnostic, pronostic et prévention d'un hématome rachidien}

L'hématome rachidien est une complication rare et potentiellement catastrophique de l'anesthésie rachidienne ou péridurale. On ne connaît pas la véritable incidence de dysfonction neurologique résultant de complications hémorragiques associées au blocage neural central. Une vaste revue des publications a permis à Tryba de trouver 13 cas d'hématome rachidien sur 850000 anesthésies péridurales et sept cas sur 650000 rachianesthésies. ${ }^{1}$ Le calcul de l'incidence qui en découle est approximativement de moins de 1 sur 150000 cas d'anesthésie péridurale et de moins de 1 sur 220000 cas de rachianesthésie. ${ }^{1}$ Ces estimations représentent la limite supérieure de l'intervalle de confiance de $95 \%$, la fréquence totale est donc beaucoup plus faible. De plus, les caractéristiques des patients et les variables anesthésiques associées à un risque accru d'hémorragie rachidienne, comparées aux valeurs de base, ont été définies par des études de cas et des séries cliniques. ${ }^{2-4}$ Ainsi, le risque associé au cathéter péridural à demeure et à l'administration biquotidienne d'héparine de bas poids moléculaire (HBPM) a été estimé à un sur 3100 patients. ${ }^{5}$ Il est utile de dépister les populations de patients à risque, mais la connaissance des techniques de gestion qui facilitent la détection et l'évaluation de nouveaux déficits neurologiques périopératoires est encore plus déterminante.

Dans le présent numéro du Journal canadien d'anesthésie, Schwarz et coll. ${ }^{6}$ présentent un cas d'hématome rachidien associé à l'administration sc standard d'héparine thromboprophylactique avec un cathéter péridural en place. Le patient a connu une récupération neurologique complète, malgré l'absence d'intervention chirurgicale. Le titre de l'article met en relief les aspects particuliers de ce cas - l'âge du patient constitue un facteur de risque potentiel et une récupération neurologique spontanée peut survenir 
malgré des déficits significatifs. Le titre démontre aussi l'idée persistante que des déficits sensoriels et moteurs sont des symptômes atypiques de l'hématome rachidien. Cette fausse idée continue de retarder l'intervention et influence l'évolution neurologique.

L'arrivée de nouveaux anticoagulants et d'antiagrégants plaquettaires, l'équilibre complexe des complications thromboemboliques et hémorragiques et les indications changeantes pour l'anesthésie/l'analgésie régionale nécessitent une approche individuelle. Espérons que l'analyse qui suit des tendances et des controverses en cours comprenant «tout ce que vous avez toujours voulu savoir au sujet de l'hématome rachidien sans jamais avoir la chance de le demander», facilitera la prise en charge des patients.

\section{Quelle est la fréquence de l'hématome rachidien spontané ?}

L'hématome rachidien, en l'absence de trauma ou d'anticoagulothérapie, survient rarement. Néanmoins, des complications hémorragiques spontanées et majeures, comme les saignements intracrâniens, intramédullaires, intraoculaires, médiastinaux ou rétropéritonéaux, ont été observés à la suite de traitement antithrombotique et thrombolytique. Les facteurs de risque comprennent l'intensité de l'effet anticoagulant, l'âge avancé, le sexe féminin, des antécédents d'hémorragie gastro-intestinale, l'usage concomitant d'aspirine et la durée du traitement. ${ }^{7}$ Pendant le traitement à la warfarine, un rapport international normalisé (RIN) de 2,0 à 3,0 est associé à un faible risque d'hémorragie : $<3 \%$ pour un traitement de trois mois. Des doses plus élevées $(\mathrm{RIN}>4)$ comportent une hausse significative des risques d'hémorragie (7\%). L'incidence de complications hémorragiques pendant l'anticoagulothérapie avec de l'héparine normale, aussi bien qu'avec de l'HBPM, est de $<3 \%$. ${ }^{7}$ Le traitement thrombolytique représente le plus grand risque de saignement, chez $6 \%$ à $30 \%$ des patients. ${ }^{8}$ Les patients qui nécessitent une anticoagulothérapie préopératoire, comme ceux qui ont des antécédents de fibrillation auriculaire ou une valvule prothétique, ne sont pas les meilleurs candidats à l'anesthésie neuraxiale. Souvent, l'effet anticoagulant n'est que partiellement renversé pour éviter des complications thrombotiques et/ou les patients reçoivent une anticoagulation postopératoire agressive. L'hémostase normale pour la mise en place d'une aiguille ou le retrait d'un cathéter n'est présente que pour un court intervalle.

\section{Quelles sont les caractéristiques des patients associées} à un risque significatif d'hématome rachidien ?

Les facteurs reliés à l'hématome rachidien et à l'hémorragie spontanée sont semblables dans le contexte d'une anticoagulothérapie - l'âge avancé et le sexe féminin. Un faible poids et une maladie hépatique ou rénale concomitante peuvent aussi exacerber la réaction d'anticoagulation et augmenter le risque théorique. Dans une série de 40 cas d'hématomes rachidiens liés à une thromboprophylaxie avec de l'HBPM, 75 \% étaient des femmes âgées. ${ }^{3}$ Le dosage de l'HBPM n'est pas ajusté au poids et l'effet anticoagulant de l'HBPM n'est pas monitoré à cause d'une biodisponibilité fiable. De plus, même si on sait que l'insuffisance rénale prolonge et accroît l'effet anti-Xa, la créatinine sérique de base n'était pas toujours mesurée avant la thromboprophylaxie avec de l'HBPM. Ces facteurs peuvent avoir contribué au nombre d'hématomes rachidiens. La pharmacologie de la warfarine est aussi significativement affectée par l'âge, la diète et l'activité du patient. La grande variabilité de la réponse du patient à la warfarine nécessite un monitorage fréquent. De toute évidence, les facteurs reliés au patient ne sont pas «contrôlables», mais ils devraient être examinés au moment de choisir une technique anesthésique régionale et l'intensité du monitorage neurologique périopératoire.

\section{Quelle est la source du saignement et combien de sang doit s'accumuler avant qu'une ischémie de la moelle épinière ne survienne ?}

On retrouve la majorité des hématomes rachidiens dans l'espace péridural à cause du plexus veineux proéminent. À l'inverse, le saignement sous-arachnoïdien qui cause des lésions neurologiques est rarement rapporté à cause de l'effet de dilution du liquide céphalo-rachidien et de l'absence de gros vaisseaux sanguins. La source réelle de l'hémorragie, artérielle ou veineuse, demeure controversée. Un saignement artériel devrait s'accumuler rapidement et causer une ischémie neurale tôt après le traumatisme. Cependant, la plupart des hématomes rachidiens deviennent symptomatiques quelques jours après la mise en place de l'aiguille ou du cathéter, non immédiatement après l'opération, ce qui laisse croire que le saignement n'est pas artériel. Par ailleurs, un saignement veineux s'accumulerait lentement, mais serait théoriquement tamponné avant de dépasser la pression de perfusion de la moelle épinière. Donc, aucun modèle ne représente complètement le scénario clinique. Le volume de sang nécessaire pour causer une ischémie médullaire varie également et dépend du site du saignement (la queue de cheval est relativement résistante, tandis que la zone limitrophe de la moelle est plus exposée), de la présence d'anomalies à la colonne vertébrale et de la rapidité avec laquelle le sang s'accumule. Fait intéressant, certains hématomes HBPM comportent moins 
de sang que la quantité normalement injectée pendant le colmatage sanguin péridural. ${ }^{3}$

\section{Comment s'installent habituellement les symptômes neurologiques?}

L'apparition postopératoire immédiate des symptômes est occasionnelle ; il est rare qu'un hématome rachidien se présente comme un blocage neuraxial "prolongé». Dans une série, l'intervalle moyen entre le début du traitement avec l'HBPM et la dysfonction neurologique était de trois jours, tandis que le temps écoulé entre l'apparition des symptômes et la laminectomie était de plus de $24 \mathrm{~h}^{3}{ }^{3}$ Moins du tiers des patients ont connu une récupération neurologique bonne ou satisfaisante. Ces résultats correspondent à ceux de Vandermeulen et coll., ${ }^{2}$ qui indiquent qu'il s'écoulait environ $15 \mathrm{~h}$ entre le début de nouveaux déficits neurologiques et la paralysie complète. De plus, on a noté que s'il y a plus de huit heures entre le développement de la paralysie et l'intervention chirurgicale, la récupération neurologique complète est peu probable. $^{2}$

\section{À quel moment devrait-on faire un examen d'ima- gerie radiographique et/ou consulter un neurochirurgien ?}

Le diagnostic différentiel des symptômes neurologiques postopératoires, nouveaux ou progressifs, comprend la neuropraxie chirurgicale, le blocage neuraxial prolongé ou exacerbé, le syndrome des artères spinales antérieures, l'exacerbation de désordres neurologiques préexistants et la présentation d'une condition neurologique non diagnostiquée antérieurement, aussi bien qu'un hématome rachidien. L'évaluation repose sur l'identification de causes réversibles ou traitables. C'est pourquoi, tout symptôme nouveau ou progressif apparaissant lors d'une analgésie péridurale justifie l'arrêt immédiat de la perfusion (le cathéter demeure in situ) pour écarter toute contribution possible de l'anesthésique local ou de l'effet de volume. Si l'anesthésique péridural est en cause, un prompt retour à la normale devrait survenir. L'évolution neurologique étant liée au diagnostic et à l'intervention précoces, il est crucial d'obtenir un examen d'imagerie radiographique, de préférence par résonance magnétique, dès que possible. Un neurochirurgien devrait être rapidement consulté aussi pour déterminer l'urgence de la chirurgie. Rappelons que tous les hématomes rachidiens ne sont pas traités par laminectomie ; la résolution spontanée des déficits a déjà été signalée. ${ }^{6,9} \mathrm{La}$ décision, d'observer $v s$ d'intervenir chirurgicalement, appartient au neurochirurgien.

\section{L'hématome rachidien est-il rapporté dans la popula- tion obstétricale ?}

Comme la grossesse et la période immédiate du postpartum sont associées à un état d'hypercoagulabilité possible, on croit généralement que les parturientes ne sont pas à risque d'un hématome rachidien. Dans la série de Vandermeulen et coll., ${ }^{2}$ cinq des 61 hématomes rachidiens concernent pourtant des parturientes. Dans deux cas, un trouble de la coagulation (thrombocytopénie, prééclampsie) était présent. Une patiente présentait un épendymome péridural non diagnostiqué antérieurement. Aucun facteur de risque n'a été constaté chez les deux autres patientes. Jusqu'à maintenant, aucun hématome rachidien n'a été associé au traitement antithrombotique du péripartum. Toutefois, il n'existe pas de grande série qui documenterait la sécurité du blocage neuraxial en présence d'anticoagulation selon les niveaux thérapeutiques nécessaires dans cette population. Le risque relatif demeure donc inconnu.

\section{Comparés aux populations chirurgicales et obstétri- cales, les patients des services antidouleur ambula- toires devraient-ils faire l'objet d'une attention particulière?}

Quelques investigations et études de cas suggèrent que les patients soumis à un bloc antidouleur chronique soient plus à risque de complications neurologiques. ${ }^{10,11}$ Dans une revue du Projet de l'American Society of Anesthesiologists sur les réclamations réglées, Cheney et coll. ${ }^{10}$ ont rapporté que les lésions médullaires étaient la principale cause de réclamation dans les années 1990 et qu'un important facteur associé était le bloc utilisé pour traiter la douleur chronique, y compris l'injection péridurale de stéroïde péridural. Dans une série de 1214 patients recevant une injection péridurale de stéroïde, incluant 383 $(32 \%)$ sous médication antiplaquettaire, ${ }^{12}$ aucun hématome rachidien n'est rapporté. Par contre, 42 patients présentaient des symptômes neurologiques ou l'aggravation de symptômes préexistants qui persistaient pendant plus de $24 \mathrm{~h}$ après l'injection. La durée moyenne des symptômes était de trois jours. On a supposé qu'un effet de pression (ischémique) de l'injection ou une réponse inflammatoire à l'un des composants, ou à plusieurs, contribuent à ces déficits neurologiques. On a recommandé d'inciter les patients à consulter un médecin si un trouble neurologique évident survenait, puisque c'est le genre de symptômes annonçant l'hématome rachidien. 
Quelles sont les options pour les patients qui ont besoin d'anticoagulothérapie ou de traitement thrombolytique d'urgence?

Les patients qui ont récemment subi une anesthésie neuraxiale et ont besoin d'une anticoagulothérapie d'urgence pour une ischémie des membres, un syndrome coronaire aigu ou une thrombose veineuse profonde/une embolie pulmonaire représentent une énigme pour les anesthésiologistes. Idéalement, le patient devrait être interrogé avant le traitement antithrombotique ou thrombolytique pour savoir s'il y a eu une ponction lombaire, une anesthésie rachidienne ou péridurale récentes ou l'injection péridurale de stéroïde afin d'administrer le traitement et le monitorage appropriés. La décision de maintenir ou de retirer un cathéter neuraxial est fondée sur le degré/la durée du traitement antithrombotique. Comme le traitement combiné (médicament antiplaquettaire avec héparine et/ou thrombolytiques) représente un plus grand risque de saignement, il faut penser à des traitements qui présentent le moins d'impact possible sur la coagulation. ${ }^{7,8}$ Malheureusement, tandis que les anesthésiologistes connaissent bien le potentiel de saignement médullaire chez ces patients, d'autres spécialistes n'ont que récemment pris connaissance du risque. Avec pour résultat que les anesthésiologistes ne peuvent être informés qu'après l'établissement d'anticoagulothérapie. Dans ce contexte, il faut s'entendre sur le moment où l'hémostase peut être restaurée pour permettre le retrait du cathéter tout en pesant les risques relatifs d'hémorragie et de thrombose. Le renversement complet de l'effet anticoagulant peut ne pas être faisable à cause du risque de complications thromboemboliques. Il est donc crucial de poursuivre la formation des cliniciens responsables d'administrer des médicaments qui affectent l'hémostase.

\section{De nouveaux agents antithrombotiques sont conti- nuellement mis sur le marché. Qu'est-ce qu'une stratégie appropriée ?}

Des nouveaux médicaments antithrombotiques qui ciblent divers stades du système hémostatique, comme l'inhibition de l'agrégation plaquettaire, le blocage des facteurs de coagulation ou la stimulation de la fibrinolyse, font toujours l'objet de recherche. Les plus largement étudiés sont des antagonistes spécifiques de récepteurs plaquettaires et des inhibiteurs de la thrombine. Beaucoup de ces antithrombotiques ont une demi-vie prolongée et ont des effets difficiles à renverser sans l'administration de composants sanguins. De plus, comme le risque de complications thromboemboliques est réduit par un traitement antithrombotique peropératoire ou postopératoire précoce, il est fort probable que ces principes vont guider la recherche de nouvelles thérapies pharmacologiques. Malheureusement, le titrage postopératoire précoce est associé à un saignement chirurgical et souvent à un saignement relié à l'anesthésie. C'est ce qui a été noté avec l'héparine normale, l'HBPM et le fondapari nux. $3,7,13$

Il ne sera sans doute pas possible d'évaluer le risque d'hématome rachidien d'après les données des phases II et III des essais cliniques sur les nouveaux médicaments. Les compagnies pharmaceutiques et la Food and Drug Administration élaborent des méthodologies pour minimiser le potentiel de complications catastrophiques de ce genre. Par exemple, pendant les tests cliniques sur le fondaparinux, le patient était exclu de l'étude si le bloc neuraxial était traumatique ou s'il avait nécessité le passage de plus d'une aiguille. ${ }^{13}$ En attendant qu'une importante documentation ne soit disponible pour préciser la sécurité des techniques neuraxiales chez les patients traités avec des antithrombotiques comme le fondaparinux, la décision de réaliser un blocage neuraxial devrait s'appuyer sur la fréquence et la sévérité des saignements chirurgicaux (comme substitut au saignement médullaire), les conditions dans lesquelles a été réalisé le bloc pendant les essais cliniques et le risque lié aux autres techniques anesthésiques. L'effet prolongé des agents antithrombotiques expérimentaux va vraiment rendre difficile le retrait du cathéter à demeure pendant l'hémostase normale. On peut penser que le cathéter neuraxial à demeure sera retiré avant le début de la thromboprophylaxie.

\section{Les blocs périphériques continus représentent-ils un «risque minimal» ?}

Quoique l'hématome rachidien soit la complication hémorragique la plus importante de l'anesthésie régionale, étant donné la nature catastrophique du saignement dans un espace fixe et non compressible, le risque associé aux suites de l'anesthésie des plexus et de l'anesthésie périphérique demeure inconnu. Il n'y a pas de recherches sur la fréquence et la sévérité des complications hémorragiques à la suite de bloc périphérique chez des patients anticoagulés. Cependant, peu d'articles signalent des complications sérieuses à la suite de la canulation d'une gaine neurovasculaire pour des indications chirurgicales, radiologiques ou cardiaques. Les quelques cas rapportés de saignement important, associé à des techniques non neuraxiales, sont survenus après un blocage de la loge du psoas ou un blocage sympathique lombaire ; une lésion neurologique n'était pas toujours mentionnée. ${ }^{14-16}$ Ces cas suggèrent que des pertes sanguines significatives (suivies de transfusion 
et même de la mort), plutôt que des déficits neuraux, puissent être la plus importante complication des techniques régionales non neuraxiales chez un patient anticoagulé. Le manque d'informations ne permet donc pas de faire des recommandations finales. On peut raisonnablement dire que des énoncés généraux sur l'anesthésie neuraxiale et l'anticoagulation peuvent s'appliquer aux techniques régionales et périphériques. ${ }^{17}$ Cependant, ce pourrait être plus restrictif que nécessaire pour des techniques qui concernent le système vasculaire superficiel et compressible.

En résumé, les facteurs de risque d'hématome rachidien incluent le patient et les variables pharmacologiques et anesthétiques régionales. Il faut toutefois se rappeler que l'identification des facteurs de risque et l'élaboration de recommandations n'élimineront pas complètement le risque d'un hématome rachidien. Dans la série de Vandermeulen, même si on a relevé des anomalies hémostatiques ou des difficultés avec les aiguilles pour ponction chez $87 \%$ des patients, $13 \%$ ne présentaient pas de facteur de risque identifiable. ${ }^{2}$ La vigilance du monitorage est critique pour permettre l'évaluation précoce d'une dysfonction neurologique et d'une prompte intervention. Nous devons mettre l'accent non seulement sur la prévention d'hématome rachidien, mais aussi sur l'optimalisation de l'évolution neurologique.

\section{References}

1 Tryba M. Epidural regional anesthesia and low molecular heparine: pro (German). Anasthesiol Intensivmed Notfallmed Schmerzther 1993; 28: 179-81.

2 Vandermeulen EP, Van Aken H, Vermylen J. Anticoagulants and spinal-epidural anesthesia. Anesth Analg 1994; 79: 1165-77.

3 Horlocker TT, Wedel DJ. Neuraxial block and low-molecular-weight heparin: balancing perioperative analgesia and thromboprophylaxis. Reg Anesth Pain Med 1998; 23(Suppl 2): 164-77.

4 Horlocker TT, Wedel DJ, Schroeder DR, et al. Preoperative antiplatelet therapy does not increase the risk of spinal hematoma associated with regional anesthesia. Anesth Analg 1995; 80: 303-9.

5 Schroeder DR. Statistics: detecting a rare adverse drug reaction using spontaneous reports. Reg Anesth Pain Med 1998; 23(Suppl 2): 183-9.

6 Schwarz SK, Wong CL, McDonald WN. Spontaneous recovery from a spinal epidural hematoma with atypical presentation in a nonagenarian. Can J Anesth 2004; 51: 557-61.

7 Levine MN, Raskob G, Landefeld S, Kearon C. Hemorrhagic complications of anticoagulant treatment. Chest 2001; 119(Suppl 2): 108S-21S.
8 Levine MN, Goldhaber SZ, Gore JM, Hirsh J, Califf $R M$. Hemorrhagic complications of thrombolytic therapy in the treatment of myocardial infarction and venous thromboembolism. Chest 1995; 108(Suppl 4): 291S-301S

9 Gerancher JC, Waterer R, Middleton J. Transient paraparesis after postdural puncture spinal hematoma in a patient receiving ketorolac. Anesthesiology 1997; 86: 490-4.

10 Cheney FW, Domino KB, Caplan RA, Posner KL. Nerve injury associated with anesthesia: a closed claims analysis. Anesthesiology 1999; 90: 1062-9.

11 Benzon HT, Wong HY, Siddiqui T, Ondra S. Caution in performing epidural injections in patients on several antiplatelet drugs (Letter). Anesthesiology 1999; 91: 1558-9.

12 Horlocker TT, Bajwa ZH, Ashraft Z, et al. Risk assessment of hemorrhagic complications associated with nonsteroidal antiiflammatory medications in ambulatory pain clinic patients undergoing epidural steroid injection. Anesth Analg 2002; 95: 1691-7.

13 Turpie AG, Gallus AS, Hoek JA; Pentasaccharide Investigators. A synthetic pentasaccharide for the prevention of deep-vein thrombosis after total hip replacement. N Engl J Med 2001; 344: 619-25.

14 Klein SM, D'Ercole F, Greengrass RA, Warner DS. Enoxaparin associated with psoas hematoma and lumbar plexopathy after lumbar plexus block. Anesthesiology 1997; 87: 1576-9.

15 Maier C, Gleim M, Weiss T, Stachetzki U, Nicolas V, Zenz $M$. Severe bleeding following lumbar sympathetic blockade in two patients under medication with irreversible platelet aggregation inhibitors. Anesthesiology 2002; 97: 740-3.

16 Weller RS, Gerancher JC, Crews JC, Wade KL. Extensive retroperitoneal hematoma without neurologic deficit in two patients who underwent lumbar plexus block and were later anticoagulated. Anesthesiology 2003; 98: 581-5.

17 Horlocker TT, Wedel DJ, Benzon H, et al. Regional anesthesia in the anticoagulated patient: defining the risks (The Second ASRA Consensus Conference on Neuraxial Anesthesia and Anticoagulation). Reg Anesth Pain Med 2003; 28: 172-97. 